\title{
Análise da percepção do estado de saúde dos idosos da região metropolitana de Belo Horizonte
}

\author{
Andysisof perception of the hedthstas of eldely from thentropditan area \\ of BdoHbrizatedity
}

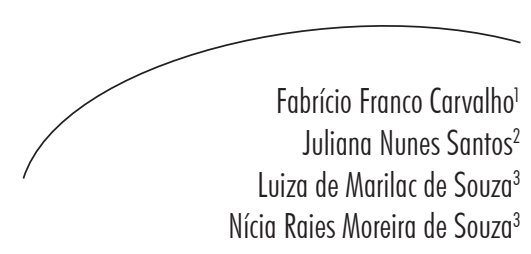

Resumo

Objetivo: Analisar a percepção do estado de saúde dos idosos da Região Metropolitana de Belo Horizonte. Método: Utilizaram-se dados da Pesquisa por Amostra de Domicílio (PAD/MG), realizada em 2009 pela Fundação João Pinheiro. Foi examinada e entrevistada amostra probabilística de 963 pessoas com idade igual ou maior a 60 anos residentes na Região Metropolitana de Belo Horizonte. A variável dependente foi a autopercepção do estado de saúde e as variáveis independentes foram: presença de doenças que exigem acompanhamento constante, tabagismo, prática regular de atividade física, trabalho, recebimento de aposentadoria e escolaridade. Foram conduzidas análises descritivas e análises de associação estatística por meio dos testes Qui-Quadrado e T Student. Resultado: Dos entrevistados, 58,2\% são do sexo feminino, com idade média de 69,4 anos e 44\% relataram o estado de saúde como sendo "muito bom e bom", e 10,7\%, "ruim ou muito ruim". Dos idosos que relataram ter problema de saúde que exigem acompanhamento constante ( $n=960), 42 \%$ possuem hipertensão arterial. Da população pesquisada, 78,5\% sabem ler e escrever um bilhete simples, $67,9 \%$ receberam aposentadoria ou pensão no mês de referência da pesquisa, $25 \%$ dos idosos relataram praticar atividade física regularmente. A autopercepção do estado de saúde se apresenta pior nos idosos que relataram presença de doenças crônicas, baixa escolaridade, menor ocupação laboral e redução das práticas de atividade física $(\mathrm{P}<0,05)$. Os resultados podem ser considerados uma ajuda na busca do desenvolvimento de ações e programas de atendimento população mineira idosa.

\footnotetext{
Faculdade de Medicina, Departamento de Fonoaudiologia. Universidade Federal de Minas Gerais. Belo Horizonte, MG, Brasil.

2 Faculdade de Medicina, Departamento de Fonoaudiologia. Universidade Federal de Minas Gerais. Belo Horizonte, MG, Brasil.

3 Centro de Estatística e Informações. Fundação João Pinheiro. Belo Horizonte, MG, Brasil.
}

Correspondência / Correspondence

Juliana Nunes Santos

E-mail: jununessantos@yahoo.com.br

\author{
Palavras-chave: \\ Envelhecimento. \\ Autoimagem. Nível de Saúde. \\ Idoso. Saúde do idoso. Belo \\ Horizonte, MG
}




\section{Abstract}

Objective: To analyze how the elderly population in the Metropolitan Region of Belo Horizonte perceive their own health situation. Methodology: Data from the Household Sample Survey (PAD/MG) were used held in 2009 by João Pinheiro Foundation. It was examined and interviewed a random sample of 963 people aged 60 years or more residents in the Metropolitan Region of Belo Horizonte. The dependent variable was self-perceived of health status and the independent variables were: diseases that require constant monitoring, smoking, regular physical activity, working, pension benefits and education. A descriptive analysis and statistical analysis of association were conducted by means of Chi-square and T Student. Results: The study interviewed 963 people aged 60 years or greater; $58.2 \%$ of the sample are women, mean age of 69.4 years and $44 \%$ reported health status as "very good or good" and 10.7\% "bad or very bad". From the subjects who reported having health problems that require constant monitoring $(\mathrm{n}=960), 42 \%$ suffer of arterial hypertension; $78.5 \%$ can be considered illiterate; $67.9 \%$ received retirement or pension in the reference month of the survey; $25 \%$ reported performing regular physical activity; 10.7\% declared their health state as being "bad or very bad". The self-perception of health status is worse among the elderly reporting chronic diseases, poor education, lack of working habits and reduced physical activity $((\mathrm{P}<0,05)$. These results can be considered as crucial information to help in the search for implementing better government funded programs to assist the elderly population of Minas Gerais.
Key words: Aging. Self Concept. Health Status. Aged. Health of the Elderly. Belo Horizonte City.

\section{INTRODUÇÃO}

O Brasil vive uma transição demográfica e epidemiológica desde as últimas décadas. ${ }^{1}$ A mudança demográfica brasileira vem acontecendo de modo abrupto, com reduções na taxa de fecundidade da população e aumento de pessoas idosas e da população em idade ativa. ${ }^{1}$ Na América Latina, entre 1980 e 2025, deverá ocorrer um aumento da população acima de 60 anos de $412 \%$. Também é previsto um aumento de idosos para 2025 em torno de 30 milhões, sendo que no ano 2000, o Brasil tinha 14 milhões de idosos. ${ }^{2}$

Estudos epidemiológicos com abordagem gerontológica ajudam não só a compreender o envelhecer, como fornecem parâmetros para organizar e praticar políticas de saúde voltadas à população de idosos em geral. ${ }^{3} \mathrm{Um}$ dos fatores mais usados em pesquisas gerontológicas é a autopercepção de saúde, pois esta antecede o declínio funcional e a mortalidade. A autopercepção do estado de saúde é um indicador global para a qual a pessoa considera, além de possíveis doenças de que seja portador, o impacto que geram no bem-estar físico, social e mental, sendo o aspecto físico o mais relevante. ${ }^{4,5}$

A autopercepção de saúde vem sendo utilizada em vários estudos, ${ }^{6,7}$ sendo considerado um método confiável, capaz de expressar vários aspectos da saúde física, cognitiva e emocional dos indivíduos. Pessoas com percepção ruim do estado de saúde têm maior risco de morte, em comparação com as que relatam uma saúde boa ou excelente. ${ }^{8}$ A percepção, portanto, mostrou ser um forte indicador de mortalidade. Além de preditor de mortalidade ${ }^{9}$, a percepção da saúde, ou autoavaliação da saúde, também está relacionada ao declínio funcional, sendo utilizada em pesquisas gerontológicas ${ }^{9-11}$

O envelhecimento da população brasileira é um dos grandes desafios a serem enfrentados. Dados preliminares do Censo 2010 mostram o alargamento do ápice da pirâmide, com o aumento expressivo da população de 60 anos e mais. ${ }^{12}$ Estima-se que, no ano de 2025, o Brasil tenha em torno de 30 milhões de pessoas com mais de 60 anos (aproximadamente 15\% da população) e será o sexto país em número de 
idosos. ${ }^{13}$ Esta nova configuração etária denota o aumento da esperança de vida ao nascer da população brasileira, a qual, segundo dados do Instituto Brasileiro de Geografia e Estatística (IBGE), foi estimada em 67,9 anos para homens e 75,5 anos para as mulheres. ${ }^{14}$

Sendo a parcela da população idosa a que mais cresce atualmente no Brasil, é de extrema importância que se conheçam as condições de saúde destes indivíduos e que se façam estudos epidemiológicos específicos para cada região, a fim de contribuir para uma gestão de saúde de acordo com as demandas locais de saúde da população pesquisada.

No Brasil, ainda são raros os estudos de base populacional, com destaque para a PNAD (Pesquisa Nacional por Amostra de Domicílios do IBGE), cuja abrangência é nacional desde $2004 .{ }^{15} \mathrm{Na}$ PNAD, investiga-se a autopercepção da saúde por meio de uma simples pergunta: "De uma maneira geral, como você considera a sua saúde?". Outras pesquisas avaliam a percepção de saúde de forma idêntica ou equivalente, já que este é um dos indicadores mais usados em pesquisas gerontológicas como preditor do declínio funcional e de outras condições de saúde e qualidade de vida dos idosos. ${ }^{4,8,9}$

O presente trabalho buscou avaliar a percepção do estado de saúde dos idosos da Região Metropolitana de Belo Horizonte e analisar as características de gênero, escolaridade, trabalho e recebimento de aposentadoria, assim como relacionar a percepção do estado de estado de saúde com a presença de doenças crônicas e hábitos de vida na população com idade igual ou superior a 60 anos de idade.

\section{METODOLOGIA}

A análise da situação de saúde da população de Minas Gerais foi investigada por meio de estudo observacional, descritivo e transversal de dados secundários, o banco de dados da PAD-MG (Pesquisa por Amostra de Domicílio) da Fundação João Pinheiro. A PAD/MG é realizada nos mesmos moldes da Pesquisa
Nacional por Amostra de Domicílios, a PNAD do IBGE. A pesquisa por amostra de domicílios de Minas Gerais, realizada no ano 2009, foi um levantamento socioeconômico baseado em amostra de 17 mil domicílios distribuídos por todo o estado, em 1.200 setores censitários de áreas urbanas e rurais de 308 municípios. A pesquisa $\mathrm{PAD} / \mathrm{MG}$ coletou informações sobre saúde, educação, trabalho, assistência social, renda e benefícios, gastos (coletivos, de alimentos e individuais) e juventude, além das características dos domicílios e dos indivíduos. ${ }^{16}$

O questionário da PAD/MG foi dividido em nove seções, a saber: características do domicílio, características dos moradores, educação, saúde, trabalho e trabalho infantil, rendimentos, gastos individuais e de domicílios e juventude. Neste estudo, ênfase será dada à análise de questões da seção "Saúde", além da análise das variáveis de identificação contidas na seção de características dos moradores.

A PAD/MG abrangeu a população residente nas unidades domiciliares - domicílios particulares e unidades de habitação em domicílios coletivos. A coleta de dados aconteceu no período de junho a novembro de 2009, utilizando-se o coletor eletrônico (Ultra Móbile Personal Computer - UMPC) para realização das operações de coleta. Os entrevistados foram pessoas contratadas pela Fundação João Pinheiro, as quais receberam treinamento teórico e prático para a realização das entrevistas domiciliares. ${ }^{16}$

$\mathrm{Na}$ Pesquisa por Amostra de Domicílios da Fundação João Pinheiro, o termo de consentimento livre e esclarecido foi substituído pelo consentimento verbal do entrevistado, obtido no momento da entrevista pelos pesquisadores da fundação. Os entrevistadores esclarecerem os moradores sobre os aspectos da pesquisa, seus benefícios, repercussões e importância na avaliação das políticas estaduais e solicitaram o consentimento dos mesmos para a participação na pesquisa. Os moradores tinham a liberdade de não aceitar e não participar da pesquisa. O índice de recusa observado está em torno de $10 \%$ a $15 \%$ das entrevistas. O estudo foi aprovado pelo Comitê 
de Ética em Pesquisa da Universidade Federal de Minas Gerais (ETIC 0347.0.203.000-10).

Com base nas respostas dos entrevistados, foi gerado um banco de dados no programa SPSS 14.0 (Statistical Package for the Social Sciences) para a PAD/MG. Neste estudo específico, foram analisadas as seguintes variáveis: autopercepção do estado de saúde, presença de doenças que exigem acompanhamento constante, tabagismo, prática regular de atividade física, trabalho, recebimento de aposentadoria e escolaridade de pessoas com idade igual ou superior a 60 anos.

As respostas às questões da $\mathrm{PAD} / \mathrm{MG}$ sobre acesso e utilização de serviços de saúde consideraram o dia 01 de junho de 2009 como data de referência. $O$ mês de maio e a semana compreendida entre os dias 24 e 30 de maio foram considerados, respectivamente, o mês e a semana de referências do estudo.

Um banco de dados específico para este estudo foi montado a partir das informações obtidas na PAD/MG. Para fins de análise descritiva, realizaram-se a distribuição de frequência das variáveis, categorias em estudo e medidas de tendência central e de dispersão das variáveis contínuas. Os dados foram previamente conferidos. Os dados inconsistentes e as informações ignoradas trataram-se adequadamente. Utilizaramse os testes Qui-Quadrado e Student para análise inferencial com valores de $\mathrm{p} \leq 0,05$. $\mathrm{O}$ processamento e a análise dos dados foram realizados pelo programa SPSS 14.0.

\section{RESULTADOS}

Em Minas Gerais, a Pesquisa de Amostra por Domicílio (PAD/MG), realizada pela Fundação João Pinheiro, entrevistou 7.526 pessoas com idade igual ou maior a 60 anos. Dessas, 963
(12,7\%) residem na Região Metropolitana de Belo Horizonte e serão foco do presente estudo.

Os aspectos gerais da população de idosos da Região Metropolitana de Belo Horizonte estudada na seção saúde da PAD/MG serão apresentados em três grupos: condições físicas (gênero, doenças crônicas e percepção do estado de saúde), condições socioeconômicas (escolaridade, trabalho e recebimento de aposentadoria) e hábitos de vida (prática de atividade física e tabagismo). Todos esses aspectos serão relacionados com a percepção do estado de saúde.

Dos entrevistados, 403 indivíduos são do sexo masculino (41,8\%). Em relação às condições físicas, destaca-se que a população idosa é predominantemente feminina $(58,2 \%)$. A idade média dos idosos foi 69,4 anos, variando entre 60 e 99 anos $( \pm 7,6)$. Dos idosos que relataram ter problema de saúde que exigem acompanhamento constante $(\mathrm{n}=960) 42 \%$ possuem hipertensão arterial. Da população com idade maior ou igual a 60 anos entrevistada pela PAD/MG na RMBH, apenas $10,7 \%$ relataram o estado de saúde como "ruim ou muito ruim".

Sobre as condições socioeconômicas, 756 entrevistados $(78,5 \%)$ sabem ler e escrever um bilhete simples. Dos entrevistados, 653 indivíduos $(67,9 \%)$ receberam aposentadoria ou pensão previdenciária pública no mês de referência da pesquisa. Em relação aos hábitos de vida saudável, apenas $25 \%$ dos idosos relataram praticar atividade física regula e $41,7 \%$ são tabagistas.

A relação entre a autopercepção do estado de saúde dos idosos residentes na Região Metropolitana de Belo Horizonte e condições físicas, socioeconômicas e hábitos de vida podem ser visualizados nas tabelas 1 e 2 . 
Tabela 1 - Relação entre a autopercepção do estado de saúde dos idosos e condições físicas, socioeconômicas e hábitos de vida, Região Metropolitana de Belo Horizonte, MG, 2009.

\begin{tabular}{|c|c|c|c|c|c|c|c|c|}
\hline \multirow{3}{*}{$\begin{array}{c}\text { Características } \\
\text { dos idosos }\end{array}$} & \multicolumn{6}{|c|}{ Autopercepção do estado de saúde } & \multirow{3}{*}{ Teste } & \multirow{3}{*}{ Valor de $\mathrm{p}$} \\
\hline & \multicolumn{2}{|c|}{$\begin{array}{l}\text { Muito bom e } \\
\text { bom }\end{array}$} & \multicolumn{2}{|c|}{ Regular } & \multicolumn{2}{|c|}{$\begin{array}{l}\text { Ruim e muito } \\
\text { ruim }\end{array}$} & & \\
\hline & $\mathrm{n}$ & $\%$ & $\mathrm{n}$ & $\%$ & $\mathrm{n}$ & $\%$ & & \\
\hline \multicolumn{9}{|l|}{ Sexo } \\
\hline Masculino & 198 & 49,5 & 168 & 42 & 34 & 8,5 & \multirow[t]{2}{*}{8,83} & \multirow[t]{2}{*}{$0,03 *$} \\
\hline Feminino & 232 & 41,5 & 258 & 46,1 & 69 & 12,4 & & \\
\hline \multicolumn{9}{|c|}{ Prática de atividade física } \\
\hline $\operatorname{Sim}$ & 131 & 54,4 & 98 & 40,6 & 12 & 5 & \multirow{2}{*}{18,8} & \multirow{2}{*}{$0,005^{*}$} \\
\hline Não & 297 & 41,7 & 326 & 45,7 & 90 & 12,6 & & \\
\hline \multicolumn{9}{|l|}{ Doença crônica } \\
\hline $\operatorname{Sim}$ & 229 & 31,9 & 386 & 53,9 & 102 & 14,2 & \multirow{2}{*}{198,6} & \multirow{2}{*}{$0,000^{*}$} \\
\hline Não & 201 & 83,4 & 39 & 16,2 & 1 & 0,4 & & \\
\hline \multicolumn{9}{|l|}{ Fuma cigarros } \\
\hline $\operatorname{Sim}$ & 59 & 54,1 & 36 & 33 & 14 & 12,9 & \multirow{2}{*}{10,1} & \multirow{2}{*}{0,11} \\
\hline Não & 62 & 40,7 & 77 & 50,7 & 13 & 8,6 & & \\
\hline \multicolumn{9}{|l|}{ Sabe ler e escrever } \\
\hline $\operatorname{Sim}$ & 365 & 48,5 & 332 & 44 & 56 & 7,5 & \multirow{2}{*}{49,5} & \multirow{2}{*}{$0,000^{*}$} \\
\hline Não & 65 & 31,6 & 94 & 45,7 & 47 & 22,9 & & \\
\hline \multicolumn{9}{|c|}{ Trabalhou na última semana } \\
\hline $\operatorname{Sim}$ & 108 & 63,5 & 59 & 34,7 & 3 & 1,8 & \multirow{2}{*}{36,8} & \multirow{2}{*}{$0,000^{*}$} \\
\hline Não & 321 & 40,9 & 364 & 46,4 & 100 & 12,7 & & \\
\hline \multicolumn{9}{|l|}{ Recebe Aposentadoria } \\
\hline $\operatorname{Sim}$ & 290 & 44,4 & 294 & 45 & 69 & 10,6 & \multirow{2}{*}{9,1} & \multirow{2}{*}{0,16} \\
\hline Não & 140 & 46,1 & 131 & 43 & 33 & 10,9 & & \\
\hline
\end{tabular}

Tabela 2 - Relação entre a autopercepção do estado de saúde e idade dos idosos residentes na Região Metropolitana de Belo Horizonte, MG, 2009. (N=959)

\begin{tabular}{|c|c|c|c|c|c|c|c|c|}
\hline & \multicolumn{6}{|c|}{ Autopercepção do estado de saúde } & \multirow{3}{*}{$\begin{array}{c}\text { Teste } \\
\text { ANOVA }\end{array}$} & \multirow{3}{*}{$\begin{array}{l}\text { Valor } \\
\text { de } \mathrm{p}\end{array}$} \\
\hline & \multicolumn{2}{|c|}{ Muito bom e bom } & \multicolumn{2}{|c|}{ Regular } & \multicolumn{2}{|c|}{ Ruim e muito ruim } & & \\
\hline & Média & $\begin{array}{l}\text { Desvio } \\
\text { padrão }\end{array}$ & Média & $\begin{array}{l}\text { Desvio } \\
\text { padrão }\end{array}$ & Média & $\begin{array}{l}\text { Desvio } \\
\text { padrão }\end{array}$ & & \\
\hline Idade & 68,7 & 7,5 & 69,6 & 7,4 & 70,8 & 8,7 & 2,27 & 0,07 \\
\hline
\end{tabular}




\section{DISCUSSÃO}

A autopercepção do estado de saúde é um importante instrumento conhecedor da situação global do indivíduo e o impacto que este gera no bem-estar físico, social e mental. Vários estudos têm analisado a autopercepção e os fatores associados à saúde, situação econômica e social como causas na mudança da percepção do estado de saúde. ${ }^{4,11,17-20 .}$

A Pesquisa por Amostra de Domicílios de Minas Gerais (PAD/MG) teve como objetivo auxiliar o monitoramento das políticas públicas no estado, por intermédio da realização de um estudo epidemiológico representativo para toda a população mineira. ${ }^{16}$ As informações referentes a condições de saúde e fatores associados investigados pela $\mathrm{PAD} / \mathrm{MG}$ são o foco do presente estudo, o qual pretende discutir a autopercepção do estado de saúde dos idosos da RMBH como importante avaliador da saúde da população.

Dos entrevistados, $8,5 \%$ dos homens e $12,4 \%$ das mulheres relataram estado de saúde ruim ou muito ruim. Estudo realizado na cidade de Florianópolis/SC mostrou que 12,8\% dos homens e 13,9 \% das mulheres têm percepção do estado de saúde ruim, ${ }^{18}$ sendo pior que os achados do presente estudo. Na cidade de Goiânia/GO, pesquisa mostrou que $6,9 \%$ dos homens e $15,2 \%$ das mulheres apresentam percepção do estado de saúde ruim. ${ }^{21}$ Em Porto Alegre, esse índice foi $7,0 \%$ nos homens e $10,4 \%$ nas mulheres, o que indica melhor percepção do estado de saúde em relação aos mineiros. ${ }^{20}$ Estudo de prevalência realizada no Brasil obteve valores semelhantes à cidade de Porto Alegre, com pobre percepção do estado de saúde em aproximadamente 6,5\% dos homens e $8,7 \%$ das mulheres acima de 60 anos. $^{17}$ O estudo de Gallegos-Carrillo (2006) aponta que a percepção do estado de saúde do México é pior do que a média brasileira, sendo que 19,8\% da população idosa referem estado de saúde ruim ou muito ruim. ${ }^{19}$ Percebem-se, portanto, diferenças regionais quanto à percepção do estado de saúde.

No presente estudo, a autopercepção do estado de saúde foi pior nas idosas da RMBH, concordando com outras pesquisas que também verificaram pior percepção de saúde no sexo feminino. ${ }^{4,9,11,17,19,21}$ Tal fato pode ser atribuído à maior prevalência de enfermidades como hipertensão, diabetes e depressão em mulheres em relação aos homens, o que lhes confere pior autoavaliação do estado de saúde., ${ }^{1,22}$ No entanto, há autores que encontraram percepções semelhantes entre os sexos estudando populações de outras localidades, ${ }^{20,23}$ o que pode ser explicado pela existência de diferenças regionais, assim como pelo tamanho e representatividade amostral, quando comparada à PAD/MG.

Com relação à idade, observou-se que o aumento da faixa etária contribuiu para a piora na autopercepção do estado de saúde, embora não tenha sido encontrada diferença estatisticamente significante $(p=0,07)$ entre os idosos com diferentes percepções do estado de saúde. Essa tendência estatística é apontada por outros autores ${ }^{19,23}$ e se assemelha a estudos ${ }^{11,20}$ que não obtiveram relação estatística.

Com o avanço da idade, os indivíduos tendem a apresentar mais problemas de saúde, como incapacidades funcionais e aumento de doenças crônicas. ${ }^{1} \mathrm{~A}$ associação entre presença de doença crônica e autopercepção do estado de saúde ruim verificada na vida dos idosos da RMBH também foi encontrada por outros autores. ${ }^{4,9,17,19,20,23}$ Atualmente, as doenças crônicas nãotransmissíveis têm sido o principal fator causal de morte na população idosa brasileira, ${ }^{12,24}$ interferindo diretamente na qualidade de vida do indivíduo e podendo ser um limitador nas atividades de vida diária, trabalho e lazer. ${ }^{12}$

No presente estudo, verificou-se relação entre a escolaridade e a autopercepção do estado de saúde dos idosos, sendo que os indivíduos que não sabiam ler ou escrever um bilhete simples, ou seja, não eram alfabetizados, apresentaram pior percepção do estado de saúde. Outros estudos apontam o mesmo achado, ${ }^{9,11,17,19,20}$ mostrando que pessoas com maior acesso à informação têm provavelmente melhores práticas de autocuidado e assistência à saúde, o que lhes confere melhor percepção do estado de saúde. Essa é também 
a concepção da OMS e do SUS brasileiro, que tem na Política Nacional de Promoção de Saúde o foco na educação da população, preparando-a para o autocuidado em saúde por meio do empoderamento de práticas de vida saudável. ${ }^{12,25}$

Os indivíduos que trabalharam na última semana do mês da pesquisa relataram apresentar melhor percepção de sua saúde do que aqueles que não possuem tal ocupação laboral. Outras pesquisas apontam o mesmo achado. ${ }^{17,19} \mathrm{~A}$ falta de atividade pós-aposentadoria acarreta piora na saúde mental dos indivíduos, influenciando a qualidade de vida negativamente. ${ }^{26} \mathrm{~A}$ inatividade no mercado de trabalho acarreta o comprometimento na capacidade funcional dos idosos, o que influencia a pior percepção do estado de saúde. ${ }^{27}$

A prática de atividade física relacionou-se à melhor percepção do estado de saúde. Outros estudos mostram que indivíduos que praticam exercícios físicos como prática de lazer possuem melhor autopercepção de saúde do que aqueles que não praticam ou realizam menos atividade física. ${ }^{11,17,20} \mathrm{~A}$ atividade física está ligada ao bom funcionamento físico e prevenção de doenças como hipertensão e doença arterial coronária. ${ }^{25}$ Consequentemente, leva à maior longevidade, $\mathrm{o}$ que confere melhor percepção de saúde aos que fazem prática regular de alguma modalidade que exija esforço físico. ${ }^{28}$

Dos entrevistados, pode-se verificar que o recebimento de aposentadoria não influenciou de maneira estatisticamente significante na percepção do estado de saúde, o que está de acordo com outros autores. ${ }^{23}$ Outros estudos apontam a importância da renda como fator influenciador na autopercepção do estado de saúde, sendo o aumento desta diretamente ligada à melhora da percepção de saúde., 9,11,17,20 Idosos com renda baixa tendem a possuir piores condições básicas de saúde. ${ }^{10}$ A renda é um importante auxiliador na procura de tratamentos e planos de saúde privado. ${ }^{29}$ Contudo, a renda de aposentadoria muitas vezes não supre as necessidades básicas dos idosos para compra de remédios, alimentos, utensílios, vestuário e lazer, fazendo com que não se torne um efeito para o aumento da percepção do estado de saúde em geral, tanto física quanto psíquica.

Não se observou associação estatística entre tabagismo e autopercepção do estado de saúde no presente estudo. $\mathrm{O}$ mesmo achado foi encontrado entre os idosos residentes em Guaramiranga, Ceará, ${ }^{23}$ mas outros estudos apontam relação direta entre tabagismo e a autopercepção negativa do estado de saúde. ${ }^{17,19,30} \mathrm{~A}$ interferência do cigarro na qualidade de vida é expressa pela perda da capacidade vital, doenças pulmonares, cardiovasculares, renais e cerebrais. ${ }^{25}$ Todavia, muitos tabagistas persistem no hábito de fumar, por apresentarem dependência pela nicotina, a qual se encontra presente juntamente com outras substâncias no tabaco.

Torna-se necessário ressaltar que este estudo é de caráter transversal, o que impossibilita estabelecer uma relação causal entre as variáveis analisadas. Existem fatores que influenciam na percepção de saúde, sendo que a presença de doenças crônicas, baixa escolaridade e ser do sexo feminino relacionam-se com uma pior percepção. Entretanto, pode-se considerar a prática de atividade física e ocupação laboral fatores contribuintes para uma melhora da percepção de saúde. $O$ estudo demonstra a necessidade de investimento governamental de atenção à saúde dos idosos para que se promova um envelhecimento saudável e de qualidade para os idosos mineiros.

A análise do banco de dados da PAD/MG permitiu identificar fatores que contribuem para o mau estado de saúde dos idosos. Os resultados podem ser considerados como uma ajuda na busca do desenvolvimento de ações e programas de atendimento a esta população mineira. Os resultados demonstraram que a saúde envolve vários aspectos, tanto orgânicos como sociais, e que desse modo deve-se entender a saúde de forma ampliada. Para tanto, são necessários novos estudos que contemplem a saúde do idoso de modo holístico, de modo que ações sejam tomadas não apenas no campo físico, mas no social e cultural, para que se possa envelhecer de forma ativa e saudável. 


\section{CONCLUSÃO}

O presente estudo analisou a situação das condições de saúde dos idosos pesquisados na RMBH por meio da utilização de banco de dados da PAD/MG. Dos idosos entrevistados, $45 \%$ relatam estado de saúde muito bom; $44 \%$, regular; e $11 \%$, ruim ou muito ruim - o que diferiu entre os sexos com pior percepção para a população feminina.

\section{REFERÊNCIAS}

1. IBGE. A dinâmica demográfica brasileira e os impactos nas políticas públicas - Indicadores Sociodemográficos e de Saúde no Brasil - 2009.

2. Veras R. Epidemiologia do envelhecimento na América Latina. In: Forlenza OV, Caramelli P. Neuropsiquiatria geriátrica. São Paulo: Atheneu; 2000. 7-21.

3. Pinto R B R, Bastos L C. Approach to research in epidemiology applied to gerontology in Brazil: literature review of papers published between 1995 and 2005. Rev Bras Epidemiol. 2007; 10(3):361-9.

4. Rigo II, Paskulin LMG, Morais EP. Capacidade funcional de idosos de uma comunidade rural do Rio Grande do Sul. Rev Gaucha Enferm 2010; 31(2): 254-61.

5. Pereira RJ, Cotta RMM, Franceschini SCC, Ribeiro RCL, Sampaio RF, Priore SE, Cecon PR. Contribuição dos domínios físico, social, psicológico e ambiental para a qualidade de vida global de idosos. Revista de psiquiatria do Rio Grande do Sul 2006; (28): 27-38.

6. Brasil. Ministério da Saúde. Secretaria de Vigilância em Saúde. Secretaria de GestãoEstratégica e Participativa. Vigitel Brasil: Vigilância de fatores de risco e proteção paradoenças crônicas por inquérito telefônico. Brasília: Ministério da Saúde; 2007.

7. Lebrão M L, et al. Saúde, Bem-estar e Envelhecimento - O Projeto Sabe no município de São Paulo. Rev Bras Epidemiol. 2005; 8(2):127-41.

8. Marcellini F. Health perception of elderly people: the results of a longitudinal study. Arch Gerontol Geriatr Suppl. 2002;8:181-9.

9. Alves LC, Rodrigues RN. Determinantes da autopercepçào de saúde entre idosos do Município de Sào Paulo, Brasil. Rev Panam Salud Publica 2005; 17(5/6):333-41.
Houve relação da percepção do estado de saúde com as variáveis: condições físicas (gênero, doenças crônicas), condições socioeconômicas (escolaridade, e trabalho) e hábitos de vida (prática de atividade física). A autopercepção do estado de saúde se apresenta pior nos idosos que relataram presença de doenças crônicas, baixa escolaridade, menor ocupação laboral e redução das práticas de atividade física.

10. Lima-Costa M F, et al.A influência de respondente substituto na percepção da saúde de idosos: um estudo baseado na Pesquisa Nacional por Amostra de Domicílios (1998, 2003) e na coorte de Bambuí, Minas Gerais, Brasil. Cad Saude Publica. Ago. 2007; 23(8):1893-902.

11. Lima-Costa MF, Firmo JOA, Uchoa E. A estrutura da auto-avaliação da saúde entre idosos: projeto Bambuí. Rev Saude Publica. 2004; 38(6):827-34.

12. Oliveira L A P. Primeiros resultados do Censo Demográfico 2010. Rev Bras Estud Popul 2011; 28(1): 3-4.

13. World Health Organization .Envelhecimento ativo: uma política de saúde / World Health Organization; tradução Suzana Gontijo. Brasília: Organização PanAmericana da Saúde, 2005.

14. IBGE . Perfil dos idosos responsáveis pelos domicílios no Brasil - 2000. Rio de Janeiro; 2002.

15. Travassos C, Viacava F, Laguardia J. Os suplementos saúde na Pesquisa Nacional por Amostra de Domicílios (PNAD) no Brasil. Rev Bras Epidemiol. 2008; 11(supl.1):98-112.

16. Fundação João Pinheiro Centro de Estudos de Política Públicas. Relatório Metodológico PAD MG / Coordenação de Nícia Raies Moreira de Souza - Belo Horizonte:2009.

17. Barros MBA, Zanchetta LM, Moura EC, Malta DC. Auto-avaliação da saúde e fatores associados, Brasil, 2006. Rev Saude Publica. 2009 43(supl.2):27-37.

18. Benedetti TB, Petroski EL, Gonçalves LT. Condições de saúde nos idosos de Florianópolis. ACM. Arquivos catarinenses de medicina. 2006; 35(1):44-51.

19. Gallegos-Carrillo K, García-Peña C, Duran-Muñoz C, Reyes H, Durán-Arenas L. Autopercepción del estado de salud: una aproximación al los ancianos en México. Rev Saude Publica. 2006; 40(5):792-801. 
20. Hartmann ACVC. Fatores associados a autopercepção de saúde em idosos de Porto Alegre [tese]. Porto Alegre: Universidade Católica do Rio Grande do Sul. PUC/RS/Gerontologia Biomédica, 2008.

21. Silva, A.C. Atividade física habitual e saúde multidimensional de idosos na cidade de Goiânia - GO [dissertação]. Florianópolis: Universidade Federal de Santa Catarina, Atividade Física relacionada à Saúde; 2005.

22. Brasil. Ministério da Saúde. Secretaria Sistema de Pactuação de Indicadores de Atenção Básica / DATASUS, Pacto pela Saúde. - Brasília: Ministério da Saúde, 2011.

23. Santos, M. A.B. Condições de vida e saúde da população idosa do município de Guaramiranga-CE [dissertação]. Rio de Janeiro: s.n; 2008.

24. Brasil. Ministério da Saúde. Secretaria de Atenção à Saúde. Departamento de Atenção Básica. Envelhecimento e saúde da pessoa idosa / Ministério da Saúde, Secretaria de Atenção à Saúde, Departamento de Atenção Básica Brasília : Ministério da Saúde, 2006.

25. Brasil. Ministério da Saúde. Secretaria de Atenção à Saúde. Departamento de Atenção Básica. Prevenção clínica de doenças cardiovasculares, cerebrovasculares e renais / Ministério da Saúde, Secretaria de Atenção à Saúde, Departamento de Atenção Básica. - Brasília : Ministério da Saúde, 2006.

26. Gonzáles FAL, Mota-Velazco G, Salas-Ro-Mero MF. Depression en jubilados no activos. Estudio de 234 casos. Revista médica.1995; 33(1):51-4.

27. Nunes MCR, Ribeiro RCL, Rosado LEFPL, Franceschini SC. Influência das características sociodemográficas e epidemiológicas na capacidade funcional de idosos residentes em Ubá, Minas Gerais. Rev Bras Fisioter. 2009; 13(5):376-82.

28. Pitanga FJG. Epidemiologia, atividade física e saúde. Revista brasileira de ciência \& movimento. 2002; 10(3):49-54.

29. Neri MC, Soares WL. Estimando o impacto da renda na saúde através de programas de transferência de renda aos idosos de baixa renda no Brasil. Cad Saude Publica. Rio de Janeiro, 2007; 23(8):1845-56.

30. Sernache FERF, Garcia RLR, Oliveira LD, Marielle RJ, Iancóski DV. Fatores associados ao tabagismo em idosos residentes na cidade de Londrina, Brasil. Revista brasileira de geriatria e gerontologia. 2010; 13(2):277- 88. 